\title{
Estratégias de intervenção precoce em recém-nascidos prematuros na Unidade de Terapia Intensiva Neonatal: uma revisão de literatura
}

A prematuridade associa-se às altas taxas de morbidade e mortalidade neonatal, principalmente quando associado ao baixo peso, menor idade gestacional e imaturidade global de vírios órgãos e sistemas. A prematuridade é, portanto, um problema de saúde pública que afeta $11,5 \%$ de nascidos vivos no Brasil, podendo levar a morte ou à internação prolongada nas Unidades
de Terapia Intensiva Neonatal. Esse período de internação, se não for bem acompanhado, pode acarretar em várias sequelas sensório-motoras. A intervençâo precoce é um conjunto de ações que irão potencializar um desenvolvimento neuropsicomotor adequado através de uma avaliação criteriosa e assistência fisioterapêutica personalizada. $\mathrm{O}$ objetivo deste estudo fo realizar uma revisão bibliográfica para identificar as principais estratégias fisioterapêuticas de intervenção precoce nos recém-nascidos prematuros em Unidade de Terapia Intensiva Neonatal. Para isso, foi realizado um levantamento nos manuais do Ministério da Saúde e em artigos publicados nos últimos dez anos, em bases de dados como Scientific Electronic Library Online (SciELO), Literatura Latina Americana em Ciência de Saúde (LILACS), Google Acadêmico, Coordenação de Aperfeiçoamento de Pessoal de Nível Superior (CAPES), Physiotherapy Evidence Database (PEDro) e PubMed entre Novembro de 2017 e Abril de 2018 utilizando as palavras-chave de acordo com a classificação dos Descritores em Ciências da Saúde (DCS): 'prematuridade', 'intervenção precoce', 'fisioterapia', 'recém-nascidos' e 'unidade de terapia intensiva' e seus correlatos na língua inglesa 'prematurity', 'early intervention', 'physiotherapy', 'newborn', e 'intensive care unit'. Foram selecionados 96 artigos, e dentre eles, 40 excluídos, pelo fato de não estarem de acordo com a temática proposta ou data fora inferior a estabelecida. É necessário estabelecer programas de intervenção precoce, através de estratégias auditivas, visuais, motoras, táteis-cinestésicas, proprioceptivas e vestibulares, pelo fisioterapêutica de forma contínua estabelecer programas de intervenção precoce, através de estratégias auditivas, visuais, motoras, táteis-cinestésicas, proprioceptivas e vestibulares, pelo fisioterapêutica de forma contínua
e sistematizada nas UTIN, associadas a ações coletivas de toda equipe multiprofissional, a fim de minimizar sequelas neuropsicomotoras, redução do tempo de internação e maior chance de sobrevivência com qualidade de vida a curto e longo prazo.

Palavras-chave: Prematuridade; Intervenção Precoce; Fisioterapia; Recém-nascido; Unidade de Terapia Intensiva.

\section{Strategies for early intervention in preterm infants in the Neonatal Intensive Care Unit: a literature review}

\begin{abstract}
Prematurity is associated with high rates of neonatal morbidity and mortality, especially when associated with low weight, lower gestational age and global immaturity of various organs and systems. Prematurity is therefore a public health problem that affects $11.5 \%$ of live births in Brazil, which can lead to death or prolonged hospitalization in the Neonatal Intensive Care Units. This period of hospitalization, if not well followed, can lead to several sensorimotor sequelae. Early intervention is a set of actions that will potentiate an adequate neuropsychomoto development through a careful evaluation and personalized physiotherapeutic assistance. The objective of this study was to perform a bibliographic review to identify the main physiotherapeutic strategies of early intervention in preterm newborns in the Neonatal Intensive Care Unit. For that, a survey was made in the manuals of the Ministry of Health and articles published in the last ten years, in databases such as Scientific Electronic Library Online (SciELO), Latin American Literature in Health Science (LLACS), Google Academic, Coordination (CAPES), Physiother Evidence Database (PEDro) and PubMed between Novic Lor 2017 and April 2018 using the keywords according to the classfi 'Physiotherapy' Evide 'prematurity, 'early interisention' 'phyis necessary to establish programs of early intervention, through auditory, visual, motor, tactile-kinesthetic, proprioceptive and vestibular strategies, by means of continuous and systematized physiotherapy in NICUs, associated to collective actions of all multiprofessional teams, in order to minimize neuropsychomotor sequelae, reduction of hospitalization time and greater chance of survival with quality of life in the short and long term.
\end{abstract}

Keywords: Prematurity; Early intervention; Physiotherapy; Newborn; Intensive Care Unit.

Topic: Fisioterapia

Reviewed anonymously in the process of blind peer.

Karoline de Sousa Ferreira

Faculdade Guaraí, Brasil

http://lattes.cnpq.br/6277176315555770

karol.sousa.ferreira@gmail.com

Jakeline Pereira Silva

Faculdade Guaraí, Brasil

http://lattes.cnpq.br/0326151923597766

marinethcarvalho@ymail.com

Daniela Maristane Vieira Lopes Maciel (iD

Faculdade Guaraí, Brasil

http://lattes.cnpq.br/0591590391105455

http://orcid.org/0000-0001-5934-0219

daniela.marciel@iescfag.edu.br
Received: 10/08/2019

Approved: 21/08/2019
Referencing this:

FERREIRA, K. S.; SILVA, J. P.; MACIEL, D. M. V. L.. Estratégias de intervenção precoce em recém-nascidos prematuros na Unidade de Terapia Intensiva Neonatal: uma revisão de literatura. Scire Salutis, v.8, n.2, p.62-75, 2018. DOI: http://doi.org/10.6008/CBPC2236$\underline{9600.2018 .002 .0007}$ 


\section{INTRODUÇÃO}

A prematuridade associa-se a altas taxas de morbidade e mortalidade neonatal, principalmente quando associado ao baixo peso, menor idade gestacional e imaturidade global de vários órgãos e sistemas (SILVA, 2017). Nas últimas décadas, mudanças progressivas foram registradas nas assistências perinatais e neonatais, garantindo uma redução das taxas de mortalidade em recém-nascidos pré-termo (RNPT), especialmente com a adoção de medidas preventivas nas gestações de alto risco como os corticosteroides, além da modernização e avanço do suporte ventilatório e uso de surfactante exógeno (FORMIGA et al., 2010; BRITTO, 2013).

Apesar deste avanço, muitas consequências negativas impactaram no processo de crescimento e desenvolvimento desses lactentes sobreviventes do evento da prematuridade (URZÊDA et al., 2009; FERREIRA et al., 2011). Uma das características principais do RNPT é a volubilidade dos sistemas de controle neurogênico e hormonal. Isto se deve ao imaturo desenvolvimento dos diferentes órgãos do corpo e, particularmente aos sistemas de controle que ainda não se ajustaram ao novo modo de vida. Conforme a idade gestacional, o peso ao nascimento e os fatores que aconteceram durante a vida intrauterina, esses RNPT podem exibir maior risco de desordens durante o período neonatal e sequelas que poderão afetar seu desenvolvimento neuropsicomotor (GIACHETTA et. al., 2010).

Essa imaturidade do Sistema Nervoso é um aspecto importante a ser considerado nesse contexto, pois muitos eventos que deveriam ainda acontecer são interrompidos através do nascimento prematuro, como aparecimento de sulcos e giros do encéfalo, proliferação e migração neural, organização e mielinização, conexões neuronais, arborização dendrítica e das terminações axonais. O neonato prematuro também apresenta órgãos em fase de desenvolvimento com morfologia e funcionalidade imaturos, além de particularidades anatômicas e fisiológicas próprias que o diferencia do recém-nascido à termo (MEDEIROS et al., 2009; URZÊDA et al., 2009; SILVA et al., 2017).

Funcionalmente, possuem maior vulnerabilidade biológica para desenvolverem icterícia fisiológica tardia, deficiência nas funções urinárias e digestivas, rins com eficiência diminuída, hipoglicemia, inaptidão de manter a temperatura corporal, função respiratória prejudicada, baixos níveis dos fatores de coagulação, fragilidade capilar aumentada e armazenamento impróprio de vitaminas e sais minerais (SILVA, 2017).

Alterações clínicas pós-natais em prematuros podem resultar em agravos, como hemorragia intra e periventricular, infecções, doenças respiratórias e cardíacas, distúrbios gastrintestinais, metabólicos, hematológicos e asfixia perinatal (MEDEIROS et al., 2009). Essas alterações, somadas ao excesso de intervenções na unidade de terapia intensiva neonatal (UTIN), podem levar a atrasos neurofuncionais em diferentes graus, perceptíveis ao longo dos primeiros anos de vida (RAMOS et al., 2009; PINHEIRO et al., 2011; SOUZA et al., 2013; SILVA, 2017).

A sobrecarga de estímulos ambientais nas UTIN como ruídos, luminosidade em excesso, constantes movimentos, interrupções dos ciclos de sono, aspirações desnecessárias (SCHAEFER et al., 2017), choro negligenciado, alarmes, rotinas e manipulações excessivas da equipe multidisciplinar são muito estressantes, 
podendo trazer repercussões clínicas e hemodinâmicas negativas na vida do RNPT, além de não contribuírem para uma boa adaptação extrauterina (TOSO et al., 2015).

Neste cenário, a assistência fisioterapêutica neonatal é primordial para diminuir os riscos de sequelas neurofuncionais, auxiliar na organização corporal e adaptação extrauterina, otimizar padrões sensoriais, potencializar a neuroplasticidade cerebral, otimizar padrão respiratório, favorecer padrões posturais adequados ao desenvolvimento motor fisiológico, minimizar stress ambiental e prevenir deformidades e contraturas (SILVA, 2017). Uma das estratégias viáveis é instituir um programa de intervenção fisioterapêutica precoce para potencializar o desenvolvimento adequado desses recém-nascidos prematuros na UTIN (OLIVEIRA et al., 2013; CASTRO et al., 2016; FORMIGA et al., 2016).

A intervenção precoce consiste no conjunto de ações que oferecem à criança experiências sensóriomotoras necessárias desde o nascimento, para potencializar um desenvolvimento neuropsicomotor adequado, através de uma avaliação criteriosa e assistência fisioterapêutica personalizada (FORMIGA et al., 2010). É necessário aproveitar a janela de tempo mais favorável para a plasticidade cerebral, e estabelecer uma intervenção protetora e/ou restauradora o mais precocemente possível (POGGIOLI et al., 2016; CAMARGO et al., 2017). O objetivo desse estudo é realizar uma revisão bibliográfica para identificar as principais estratégias fisioterapêuticas de intervenção precoce nos recém-nascidos prematuros em unidade de terapia intensiva neonatal.

\section{MATERIAIS E MÉTODOS}

Trata-se de um estudo de revisão teórica, a partir de um levantamento nos manuais do Ministério da Saúde e em artigos publicados nos últimos dez anos, em bases de dados como Scientific Electronic Library Online (SciELO), Literatura Latina Americana em Ciência de Saúde (LILACS), Google Acadêmico, Coordenação de Aperfeiçoamento de Pessoal de Nível Superior (CAPES), Physiotherapy Evidence Database (PEDro) PubMed, entre Novembro de 2017 e Abril de 2018, utilizando as palavras-chaves de acordo com a classificação dos Descritores em Ciências da Saúde (DCS): prematuridade, intervenção precoce, fisioterapia, recém-nascido, unidade de terapia intensiva e seus correlatos na língua inglesa prematurity, early intervention, physiotherapy, newborn, intensive care unit. Foram selecionados 96 artigos, onde 42 foram excluídos pelo fato de não estarem de acordo com a temática proposta, serem realizados fora da UTIN ou apresentarem data inferior a estabelecida.

\section{DISCUSSÃO TEÓRICA}

\section{Intervenção Precoce}

O uso das avançadas tecnologias de terapia intensiva, contribuíram com significativos avanços na área neonatal, consentindo maior sobrevida aos RNPT, contudo estes benefícios podem gerar efeitos secundários, muitas vezes negativos. Imediatamente após o nascimento, o RNPT já é encaminhado a uma UTIN e exposto a estímulos desagradáveis, como ruídos, dor e luzes intensas, ocorrendo uma mudança brusca para o RN, a separação dos pais poderá afetar tanto o desenvolvimento psíquico e físico do RN quanto 
dos próprios pais, afetando toda a relação iniciada durante a gestação, devido a sentimentos angustiantes e estressantes (LINO et al., 2015).

A fisioterapia vem agindo de maneira significativamente satisfatória, diminuindo o período de internação, preservando e recuperando a funcionalidade dos RNPT, porém necessita ser iniciada precocemente, logo após a estabilização dos parâmetros hemodinâmicos e clínicos do paciente (EBSERH, 2016), pois quanto mais tarde iniciar o tratamento maior risco de atraso no desenvolvimento motor e sensorial com prejuízos de noção espacial, percepção, esquema corporal, déficit de atenção e dificuldades cognitivas (SILVA et al., 2017).

A intervenção fisioterapêutica precoce deve ser individualizada, criteriosamente indicada e executada por profissionais capacitados em cuidados com o recém-nascido (SEGURA et al., 2010), tendo como objetivo detectar, diminuir e se possível reverter o atraso no desenvolvimento neuropsicomotor e respiratório do prematuro, através de programas que ofereçam uma sequência de estímulos, facilitem conquistas de habilidades e promovam experiências e aprendizados sobre os padrões motores apropriados para os primeiros anos de vida (SILVA et al., 2017).

Antes de iniciar qualquer tipo de intervenção, o neonato deverá ter uma capacidade mínima de controlar suas funções fisiológicas basais e responder aos estímulos do meio ambiente (ALMEIDA et al., 2014). Portanto, é imprescindível compreender que a quantidade de estímulos proporcionados está diretamente relacionada à sua capacidade, possibilidades e interesses, por isso não necessita forçar o RNPT, nem deixá-lo cansado, pois a eficácia dessa relação é estar ciente de suas capacidades e da medida exata de estímulos necessários para supri-las (FORMIGA et al., 2010).

Através da intervenção precoce a fisioterapia pode promover ainda um significativo resultado na redução ou alívio da dor, corrigir posicionamentos inadequados e dá orientações aos pais em relação ao manejo e estímulos corretos a serem realizados, estes envolvem intervenção auditiva, visual, tátil, proprioceptiva e vestibular, por meio de atividades motoras precoces (SILVA et al., 2017).

\section{Estratégias Auditivas}

A audição é a função sensorial que permite a normal aquisição e desenvolvimento da linguagem oral e a produção da fala, compreendendo as habilidades como atenção sonora, localização sonora, síntese binaural, figura-fundo, separação binaural, memória, discriminação, fechamento, ordenação temporal e resolução temporal (BRASIL, 2016).

A audição tem um importante papel no desenvolvimento de uma criança, pois é responsável por uma melhor relação do indivíduo em uma sociedade, onde a comunicação oral é predominante. Alterações auditivas resultam em déficits na linguagem e no desenvolvimento intelectual, cultural, cognitivo e social (CASALI et al., 2010).

Os sons desorganizados e alterados existentes nas UTIN são considerados ruídos e podem causar lesões físicas, alterações psíquicas e comportamentais. Estudos mostram que altos níveis de som pode induzir instabilidade fisiológica em lactentes, incluindo flutuações na frequência cardíaca, pressão arterial, perfusão 
e saturação de oxigênio, aumento da pressão intracraniana, alterações nos níveis de corticosteroides (BRASIL, 2011), hipóxia, dilatação pupilar, vasoconstrição sistêmica, aumento do consumo de oxigênio e de gasto calórico e a longo do prazo também pode acarretar um retardo no ganho de peso (PEIXOTO et al., 2010).

Na UTIN os altos níveis de ruídos dificultam a manutenção de estados de sono e um adequado desenvolvimento do SNC. Durante 48 horas de observação em uma UTI foram registrados 4.994 picos de ruídos, $86 \%$ entre 65 e $74 \mathrm{~dB}(\mathrm{~A})$ e $90 \%$ relacionados com aspectos da atividade humana. Isso corresponde a 104 picos por hora ou 1,7 por minuto. Os ruídos de aparecimento súbito são os que mais incomodam devido à propriedade comum à fibra nervosa auditiva de sempre disparar no início de um som (BRASIL, 2011).

Em decorrência desse ambiente ruidoso e medicações ototóxicas o índice de deficiência auditiva em prematuros é alto, a prevalência de alterações auditivas são maiores em crianças que permaneceram por tempo prolongado na UTI, portanto, o quanto antes for diagnosticado a perda auditiva melhor, para evitar sérias consequências no desenvolvimento infantil, afetando funções sociais, acadêmicas, cognitivas, ocupacionais e principalmente linguagem e fala (BOTELHO et al., 2008).

A musicoterapia é uma estratégia de intervenção precoce na UTIN podendo trazer benefícios tanto para o lactente quanto para sua mãe, contribuindo com redução dos níveis de estresse, avaliados com expressões faciais de prazer (vocalização, sorriso, sucção, reflexo cócleo palpebral), ganho de peso, melhora a saturação de oxigênio, regulação da frequência cardíaca, respiratória e temperatura corporal (OLIVEIRA et al., 2014).

Algumas canções de ninar gravadas podem ser oferecidas ao RNPT na incubadora, a partir de 28 semanas de idade gestacional. E canções de ninar dirigidas a lactentes realizadas ao vivo, podem ser combinadas com estimulação multimodal (intervenções auditivas, táteis, visuais e vestibulares) podendo ser iniciadas às 32 semanas de idade gestacional. Canções de ninar são composições musicais simples que os lactentes podem diferenciar claramente, caracterizando-se por um tom mais baixo e um ritmo mais lento, sendo usados e reconhecidos em várias culturas (ARNON, 2011).

A musicoterapia foi avaliada no ensaio clínico randomizado de MORAN et al., em 2015, com 26 prematuros, demonstrando efeito benéfico durante e após o procedimento de fisioterapia respiratória em RNPT na UTIN. Níveis seguros de sons dentro da UTIN devem ser preconizados, o som ambiente deve ser reduzido ao mínimo possível e não ultrapassar um som contínuo de 50 decibéis (dB), um som contínuo de 55 dB, e um Limiar máximo de 1 segundo de duração menor que $70 \mathrm{~dB}$, todos em escala $\mathrm{A}$ (resposta lenta). Esses níveis podem ser alcançados através de medidas simples como, silenciar o alarme do monitor, fechar as portas e lembrar aos pais e a equipe médica de que devem manter a voz baixa. Dessa forma, a música pode ser exercida em um volume médio confortável, sem causar estados de hiperalerta ou outros efeitos colaterais (ARNON, 2011).

A aplicação da musicoterapia é recomendada na frequência de 55 a 70 decibéis, o que pode se associar à melhora do estado comportamental e contribuir para a diminuição das frequências cardíaca e 
respiratória, maior ganho de peso, aumento da saturação de oxigênio, melhor sucção e diminuição do tempo de permanência hospitalar (ARNON et al., 2011).

No entanto, o estudo de SILVA e colaboradores, em 2013, mostrou não haver relação imediata da musicoterapia com estabilização hemodinâmica de RNPT na UTIN. Realizaram um ensaio clínico não controlado com 12 RNPT com média de idade gestacional de 34 semanas, avaliando os parâmetros de frequência cardíaca, frequência respiratória, saturação de oxigênio, pressão arterial e temperatura corporal.

Receberam 2 sessões de musicoterapia dentro da incubadora por 3 dias consecutivos, durante 15 minutos, uma hora após a mamada. As músicas selecionadas eram clássicas, de Mozart e não ultrapassaram 55 decibéis, mantendo amplitude baixa, ritmo simples e direto e frequência de aproximadamente 60 a 70 batidas por minuto. $\mathrm{O}$ aparelho de som foi posicionado do lado de fora da incubadora, em frente à portinhola mais próxima da cabeça do recém-nascido. Durante a sessão, as portinholas permaneceram abertas para que os autofalantes pudessem ser posicionados de forma correta, do lado de fora das mesmas. As evidências em relação aos reais benefícios que a musicoterapia pode trazer aos recém-nascidos prematuros ainda são controversas.

Algumas estratégias de estímulo à audição são preconizadas pelo Ministério da Saúde para minimizar a questão dos ruídos na UTIN que vão desde estrutura física a educação comportamental dos profissionais que trabalham na UTI, destacando: utilizar equipamentos com menor produção de ruído e observar pias, chão, ar condicionado; modificação de rotinas da unidade, que podem ser ruidosas, tais como discussão de casos ou passagem de plantão perto dos bebês; incentivar adoção de rotinas como a 'Hora do Psiu' (horários de uma hora e meia de duração de tranquilidade no ambiente de UTIN); adequar o manuseio de equipamentos da incubadora e do cuidado com bebê para produzir o menor ruído possível; educar continuamente toda a equipe, incluindo pessoal de suporte (limpeza, laboratório, RX, recepção, entre outras); usar estratégias para informar e obter a participação das famílias (BRASIL, 2011).

A audição também pode ser estimulada através de localizações sonoras, exercitando a memória, atenção e repetição de sons de forma lúdica, possibilitando a repetição vinda da criança aprimorando também a futura articulação da fala. Os sons emitidos pela criança são bastante variados e ricos, por isso devem ser exercitados os movimentos de boca e lábios constantemente (BARATA et al., 2010).

\section{Estratégias Visuais}

A visão é responsável por captar $80 \%$ das informações que são agregadas a outros sistemas e é a base para a aquisição das habilidades motoras (GONÇALVES et al., 2014), atividades perceptivas e mentais (SOUZA et.al., 2010). As expressões faciais, os gestos e o contato ocular também tem um importante papel na comunicação e interação social do recém-nascido. A experiência visual nos primeiros estágios da vida participa do processo de formação e maturação dos circuitos corticais, que permitirão um desenvolvimento adequado das funções visuais. A atividade neural proveniente da periferia sensorial deve ser oferecida no período de maior plasticidade cerebral, caso esta não seja apropriada, os circuitos neuronais permaneceram imaturos (PEREIRA et al., 2014). 
O desenvolvimento motor também é estimulado através da visão, proporcionando a interação com o meio, facilitando as experiências motoras e regulando o tônus muscular para dar suporte sensório-motor. Portanto, a intervenção é essencial nos primeiros anos de vida, pois estimulará o desenvolvimento global do lactente (GONÇALVES et al., 2014).

O RNPT na UTIN vivencia uma experiência visual muito diferente daquele nascido a termo que recebe alta e vai para o ambiente domiciliar. Uma dessas diferenças estabelecidas é o excesso de iluminação no ambiente das UTIN, podendo causar aumento da atividade motora e taxas metabólicas, distúrbios do sono, alteração do ritmo do ciclo circadiano e prejuízo ao ganho de peso (PICKLER et al., 2013).

Algumas estratégia foram utilizadas ao longos do tempo na tentativa de minimizar o excesso de interferência da iluminação na UTIN são elas: cobrir as incubadoras com tecido, mas quase sempre ocorre a incidência de luz nos olhos do bebê durante o seu manuseio e, em $22 \%$ das vezes, houve, nesse instante, queda significativa da saturação de oxigênio em bebês pré-termo de 26 a 37 semanas.

Além disso, reduzir o nível de iluminação mas manter o RNPT no escuro pode privá-lo de informações do ciclo dia/noite; o mais recomendado por especialista é a utilização dos ciclos dia/noite, pois estudos mostraram a presença mais precoce do ritmo circadiano em relação aos cuidados na semiescuridão contínua; atualmente vivencia-se uma fase de revolução no design das unidades neonatais, mas o meio ambiente visual ótimo para os RNPT ainda está sendo definido (BRASIL, 2011).

Uma estratégia para estimular o recém-nascido a otimizar a sua estimulação visual foi experienciada no ensaio clínico randomizado de PEREIRA et al. (2014), onde selecionou 19 RNPT entre 30 e 36 semanas, adequados para a idade gestacional, com tempo de internação maior que 10 dias, não intubados, internados em incubadoras e sem doenças congênitas ou neurológicas. Os RNPT foram divididos aleatoriamente em dois grupos, o grupo estimulação (GI) recebeu estimulação visual durante 14 dias, uma vez por dia por 5 minutos, e os participantes do grupo-controle receberam apenas duas únicas avaliações, com intervalo de 15 dias entre elas.

O RNPT era colocado semissentado na incubadora para ampliar seu campo visual e apresentado cartões com imagens simples e complexas em preto e branco, a uma distância de $30 \mathrm{~cm}$ do campo visual, numerados de um a três e aplicados de forma aleatória e não consecutiva. Este programa de estimulação visual mostrou-se eficaz somente no grupo GI por receberem estímulos diários, conseguiram captar a atenção ao estímulo, fixando o olhar e evoluindo para o movimento.

A intervenção visual não necessita de algo muito complexo, porém é necessário acontecer desde os primeiros dias de vida, no dia a dia e nos contatos afetuosos com o cuidador. Para realizar a intervenção é preciso criar experiências agradáveis, através de brincadeiras lúdicas chamando a atenção da criança. Para isso é necessário utilizar brinquedos e objetos adaptados às necessidades de cada indivíduo, objetos de diversos tamanhos e tipos, com cores de alto contraste e coloridos, brinquedos sonoros, evitar luz direta nos olhos da criança (BRASIL, 2013).

Respostas visuais de perto são recuperadas de uma forma melhor, solicitando a realização da intervenção inicial numa esfera visual mais próxima, distanciando de acordo com as respostas de melhora da 
criança, respostas essas que podem ser observadas através da fixação do olho no objeto, interesse e capacidade de exploração do estímulo (BRASIL, 2016).

\section{Estratégias Motoras}

O desenvolvimento motor é um processo de transformações no desempenho de habilidades relacionadas à idade do paciente, onde acontecem mudanças complexas e interligadas através dos aspectos de crescimento e amadurecimento dos aparelhos e sistemas do organismo, dos quais não dependem somente do processo de maturação do SNC, mas sim de aspectos biológicos, do comportamento e do ambiente em que vivem (PINTO et.al., 2008).

Este desenvolvimento acontece em etapas, que vai de movimentos rudes aos mais aperfeiçoados, evoluindo em uma sequência que se inicia através do controle da cabeça, dando seguimento ao rolar, sentar, se manter em posição ortostática e deambular. O surgimento ou não dessas aquisições motoras de acordo com cada idade são indicativos muito importantes para a deteç̧ão precocemente de possíveis anormalidades no desenvolvimento da criança (ALMEIDA, 2012).

Para que haja uma intervenção motora de qualidade, é necessário antes de tudo fazer uma avaliação criteriosa e completa do bebê, onde irá revelar a presença ou ausência de reflexos ou reações posturais de acordo com seu desenvolvimento motor, indicando se há ou não um atraso, analisando se há necessidade da intervenção precoce e adequando um acompanhamento terapêutico caso haja um atraso diagnosticado (ALMEIDA et al., 2008).

A intervenção das funções motoras ocorrerá através da abordagem proprioceptiva, proporcionando a sensação de onde se localizam partes do corpo, no espaço com maior diversidade de experiências sensoriais/sensitivas e promoção de praxias do sistema sensório motor oral e do próprio toque, resultando na consciência do próprio corpo e inibição de movimentos inalteráveis (BARATA et al., 2010).

A fisioterapia neonatal baseia-se na cinesioterapia, interação sensorial, posicionamento terapêutico, facilitação proprioceptiva e neuromuscular, que colaboram para o desenvolvimento e crescimento do neonato e são característicos para reorganização do tônus global, inibição dos padrões de postura, movimentos anormais, estimulação proprioceptiva, ampliação do limiar de sensibilidade cinestésica e tátil e adaptação do comportamento autorregulatório, prevenindo possíveis anormalidades musculoesqueléticas iatrogênicas (ALMEIDA, 2012).

O posicionamento adequado beneficia a flexão normal e orienta a linha média, promovendo o estado de organização dos bebês, seus principais objetivos são: promover a adaptação ao meio extrauterino, melhorar a estabilidade fisiológica e a organização neurocomportamental, promover a flexão postural, manter o alinhamento articular, facilitar a colocação das mãos na linha média, estimular a visão, prevenir assimetrias posturais e o desenvolvimento de padrões posturais anormais, facilitar o desenvolvimento do controle da cabeça, encorajar o desenvolvimento das habilidades motoras, promover a interação familiar e o alinhamento biomecânico. 
Nesse aspecto, é imprescindível o uso de materiais como fraldas de tecido, toalhas e lençóis. Recomenda-se que os manuseios durem de 10 a 15 minutos, visando prevenir o excesso de manipulação, sendo realizadas de acordo com a necessidade e possibilidade de cada criança, adaptando os estímulos à tolerância do recém-nascido de acordo com seu comportamento (ALMEIDA et al., 2014).

Os cuidados com o posicionamento dos RNPT ou de baixo peso na UTIN ajudam a melhorar o quadro clínico do paciente e fornecem adequados estímulos para o correto desenvolvimento motor. Por esse motivo, deve-se posicionar o RN de forma adequada, utilizando rolos de fraldas ou cobertores ao seu redor para que ocorra a manutenção do tônus muscular mais adequado, facilitando padrões normais de movimentos, diminuindo contraturas e deformidades, dando ao recém-nascidos mais conforto e segurança, além de ser uma fonte de estimulação tátil (THEIS et al., 2016).

Destaca-se a importância do posicionamento adequado do RNPT na UTI, em especial os que estão sob intubação endotraqueal, onde devem permanecer com a cabeceira elevada no leito. O posicionamento adequado não depende apenas do fisioterapeuta, mas sim, de toda a equipe multidisciplinar, objetivando prevenir as futuras deformidades articulares e complicações musculoesqueléticas (FLORES et al., 2009).

O posicionamento terapêutico admite o desenvolvimento de respostas adaptativas, semelhantes às que aparecem nos recém-nascidos a termo saudáveis, promovendo a regulação do estado neurocomportamental, proporcionando suporte postural e de movimento, facilitando a participação da criança nas expectativas sensório-motoras normais, otimizando o desenvolvimento musculoesquelético e o alinhamento biomecânico, diminuindo os efeitos iatrogênicos causados pela permanência prolongada dos RNPT na UTI neonatal (DUARTE et al., 2011).

A posição prona beneficia a postura flexora das extremidades do RN, fazendo com que ele permaneça em sono profundo por mais tempo, diminua o choro e a desorganização, ajuda o movimento antigravitacional dos extensores da cabeça, facilitando o acesso da mão à boca, além de melhorar o sincronismo torocoabdominal (NICOLAU et al., 2009), aumentar o volume corrente e melhorar a função diafragmática (ALMEIDA et al., 2014). Porém, esta posição é contraindicada para neonatos ou crianças com quadro de distensão abdominal grave, pós-operatórios imediatos de cirurgias cardíacas ou abdominais, ou em qualquer outra situação que possa causar desconforto ao paciente (JESUS et al., 2009).

O estudo de intervenção realizado por Olmedo e colaboradores com 20 RNPT, com idade gestacional entre 24 e 36 semanas, compararam as respostas fisiológicas em RNPT submetidos ao método canguru e a posição prona. O resultado demonstrou que as técnicas aplicadas foram efetivas na melhora da $\mathrm{FR}, \mathrm{SatO}_{2} \mathrm{e}$ FC pelo menos até 60 min após a aplicação das mesmas, sendo mais evidentes na posição prona.

O posicionamento em supino é o mais utilizado nas UTIs neonatais por proporcionar a maior visualização do paciente e facilitar o posicionamento dos diversos equipamentos, sendo a alternativa mais viável em casos de pós-operatórios de cirurgias torácicas e abdominais (JESUS et al., 2009). Esse posicionamento proporciona uma maior contenção postural, facilitando a flexão e os movimentos amplos dos membros superiores e inferiores, fortalecendo a rotação da cabeça e estimulando a exploração visual, sendo de todas as posturas a que menos favorece o RNPT (NICOLAU et al., 2009). 
O decúbito em supino reduz a incidência da síndrome da morte súbita, porém acarreta o atraso das aquisições motoras, favorece a hiperextensão cervical, dificulta o movimento de alcance, predispõe à obstrução do retorno venoso cerebral quando a cabeça cai para o lado, favorece a rotação da cabeça, principalmente para o lado direito, ocasiona assimetria na região occipital e favorece a postura assimétrica (DUARTE et. al., 2011). A persistência por tempo prolongado nesta postura acarreta deformidades posturais como encurtamento da cadeia muscular posterior, retração dos ombros e a abdução e rotação externa dos membros superiores e inferiores (JESUS et al., 2009).

\section{Estratégias Táteis- Cinestésicas}

A intervenção tátil-cinestésica pode ser um instrumento não farmacológico, de baixo custo, com terapia não invasiva de fácil aplicação e que contribui para o progresso dos prematuros que precisam ficar por mais tempo no ambiente da UTI. Esta intervenção se remete a uma terapia onde o toque é feito de forma suave e ordenado, baseando-se no sentido precoce do tato que já apresenta no feto a partir de sete semanas e meia de gestação, este aprimora a avanço do bebê diminuindo o tempo de internação, aumenta o ganho de peso, e reduz os episódios de apneias, promovendo um maior vínculo afetivo com os pais. A melhor resposta ao estimulo tátil dos recém-nascidos são nos seus primeiros anos de vida, quando se compara com as outras categorias sensoriais (NUNES, 2015; FIGUEIREDO et al., 2011).

Os estímulos táteis surgem de distintas intensidades de pressão sobre a pele, ativando receptores nervosos cutâneos, transformando-se em reações eletroquímicas que são levadas ao corno posterior da medula e em seguida ao hipotálamo, o estímulo doloroso é conduzido através do estímulo tátil que chega mais rápido à medula pelo fato de suas fibras serem mais mielinizadas do que as nervosas, também há liberação de endorfinas e encefalinas quando o hipotálamo é estimulado, estes hormônios têm ação semelhante à da morfina, provocando analgesia e gerando sensação de prazer, de modo que o hipotálamo é o sistema intercessor de emoções e regulador das funções viscerais (RAMADA et al., 2010).

Deve-se ter cuidado com o toque, ele precisa ser suave e não pode ser muito prolongado, porque a pele do RNPT é bastante frágil e pode ser agredida com o excesso de manipulação, é importante ressaltar que o fisioterapeuta precisa respeitar a clínica do recém-nascido, pois em alguns casos instáveis a estimulação não será viável, podendo causar hipoxemia, bradicardia e irritabilidade. Atividades de intervenção tátil utilizando objetos de diferentes cores, ruídos e texturas, são de fácil execução e obtenção pela família e tem uma grande importância para auxiliar no desenvolvimento, as atividades que promovem a interação e reforçam os laços afetivos, como a Massagem Shantala, também podem, e devem ser utilizadas no programa de intervenção precoce (BRASIL, 2016).

Outra estratégia tátil-cinestésica desenvolvida para o tratamento dos RNPT foi o Método Canguru (MC), que tem como objetivo melhorar os cuidados prestados ao RNPT, reduzindo o tempo de permanência hospitalar. No MC existe um posicionamento específico para o RN, que é envolvido e amarrado em uma faixa ou manta junto ao tórax dos pais, em posição vertical, permitindo e mantendo o contato materno (BRASIL, 2011). 
O MC facilita o processo de ganho de peso, o bebê recebe maior estimulação tátil, cinestésica, vestibular, visual, auditiva e térmica, podendo alimentar-se em livre demanda através do contado direto com a mãe. Tudo isso, simula o meio intrauterino, possibilitando o RNPT completar sua idade gestacional em um ambiente rico em estímulos e seguro, fundamental para o amadurecimento cerebral (SILVA et al., 2013).

\section{Estratégias Proprioceptivas e Vestibulares}

Durante sua permanência na UTIN, o RNPT recebe poucos estímulos vestibulares, além do risco de prejuízo a função vestibular pelo uso de antibióticos aminoglicosídeos (gentamicina e amicacina). Por isso, estimulação vestibular suave pode ajudar a consolar o RNPT nas UTIN e auxiliá-lo no despertar e na manutenção do alerta (por intermédio das conexões vestíbuloculares). Movimentos mais lentos tendem a aquietar o bebê e os mais rápidos, algo irregulares, favorecem o despertar. A estimulação vestibular está presente também durante o manejo canguru e, em todas as situações, deve ser sempre adequada às respostas exibidas pelo bebê (BRASIL, 2011).

Dentre os vários recursos de intervenção precoce proprioceptiva e vestibular destaca-se a 'redinha', que é o uso de pequenas redes dentro ou fora das incubadoras, facilitando o posicionamento do RNPT. As redinhas proporcionam aconchego dos recém-nascidos e estimula os sentidos amadurecendo os reflexos primitivos, além de proporcionar estímulo vestibular, proprioceptivo, reações de equilíbrio, integração sensorial, proteção, reorganização comportamental e tônica característica do útero materno (GOMES et al., 2014).

O RNPT através do uso da 'redinha' tem apresentado redução da irritabilidade, menor perda de gasto de energia e calor, contribuindo para o ganho de peso, além disso, observou-se nessa técnica o relaxamento do RNPT, com melhora no comportamento, pois o aconchego diante da postura uterina de flexão favorece estas respostas positivas. Além de oferecer estimulação sensorial adequada (estímulos auditivos, táteis e visuais) por meio do conforto e balanço, melhora a saturação de oxigênio e frequência cardíaca, colaborando para o desenvolvimento neuropsicomotor do RN prematuro (LINO et. al., 2015).

Outra técnica bastante utilizada nessa modalidade de intervenção é o banho de balde ou ofurô, procedimento de modo rotineiro e empírico para socialização da assistência nas UTIN brasileiras. Essa conduta é indicada e baseada no argumento de que o banho do RNPT em água aquecida é possível auxiliar na diminuição de perda de peso, tempo de internação e estresse, propiciando um resultado superior aos cuidados neonatais (ATAÍDE et al., 2016). Alguns estudos indicam que técnicas de banho neonatal humanizado podem ser benéficas no cuidado de recém-nascidos prematuros em unidade neonatal quanto a respostas fisiológicas e comportamentais (SILVA et al., 2017).

Essa técnica é uma adaptação do método que foi desenvolvido em 1997 na Holanda, por enfermeiros e obstetras para representar ao RNPT as sensações e os estímulos vivenciados no útero da mãe, com intuito de promover relaxamento, limite corporal, segurança e beneficiar a organização sensorial (ATAÍDE et al., 2016). Em um balde com água morna o recém-nascido é mergulhado até a altura dos ombros e é movimentado delicadamente por meio da flutuação assistida. $O$ terapeuta usa a ação da força de empuxo 
do meio aquático para beneficiar a propriocepção, mobilização articular, movimentação rítmica e espontânea, propriocepção, organização postural e/ou alongamento muscular do recém-nascido (SILVA et. al., 2017). As técnicas de banho promovem a melhora do comportamento, da qualidade de sono e diminuição da angústia, da dor e do choro (VIGNOCHI et al., 2010).

Um estudo longitudinal quantitativo realizado por Silva et al. (2017), com 30 RNPT, entre 27 e 35 semanas de idade gestacional, avaliou variáveis fisiológicas associadas à hidroterapia no balde, em duas sessões de 10 minutos, em dias alternados, com três avaliações sendo 15 minutos antes, imediatamente após o banho e 30 minutos depois. Evidenciando diminuição da frequência cardíaca e assegurando ser uma prática segura para parâmetros fisiológicos e ganho de peso corporal em recém-nascidos prematuros clinicamente estáveis.

\section{CONSIDERAÇÕES FINAIS}

O nascimento prematuro é um problema grave de saúde pública, sendo o principal responsável pela elevada morbidade e mortalidade neonatal. A contribuição tecnológica sem dúvidas é responsável pela maior sobrevida dessa população, mas para melhorar sua qualidade de vida, é necessário estabelecer programas de intervenção precoce pelo fisioterapêutica de forma contínua e sistematizada nas UTIN, associadas a ações coletivas de toda equipe multiprofissional. Com o objetivo de minimizar sequelas neuropsicomotoras, redução do tempo de internação e maior chance de sobrevivência com qualidade de vida a curto e longo prazo. Sugerimos mais estudos clínicos utilizando estas estratégias fisioterapêuticas de intervenção precoce, para fortalecer a atuação do fisioterapeuta baseada em evidências.

\section{REFERÊNCIAS}

ALMEIDA, C. S.; PAINES, A. V.; ALMEIDA, C. B.. Intervenção motora precoce ambulatorial para neonatos prematuros no controle postural. Revista Ciência \& Saúde, Porto Alegre, v.1, n.2, p.64-70, 2008.

ALMEIDA, G. S. N.. Desenvolvimento Motor e percepção de competência motora na infância. Dissertação (Mestrado em Reabilitação Psicomotora) - Universidade Técnica de Lisboa, Lisboa, 2012.

ALMEIDA, S. K. A.; MEJIA, D. P. M.. Os benefícios da estimulação motora em recém-nascidos na UTIN: exercícios e posicionamento terapêuticos. Monografia (Pós-graduação em Fisioterapia Hospitalar) - Faculdade SulAmericana, Goiânia, 2014.

ARNON S.. Intervenção musicoterápica no ambiente da unidade de terapia intensiva neonatal. Jornal de Pediatria, Rio de Janeiro, v.87, n.3, p.183-185. 2011. DOI: http://doi.org/10.2223/JPED.2091

ATAÍDE, V. P.; BARBOSA, J. S. V.; CARVALHO, M. G. S.; NEVES, S. M. S. G.; SANCHEZ, F. F.; GONÇALVES, R. L.. Ofurô em recém-nascidos pré-termo de baixo peso: relato de experiência. ASSOBRAFIR Ciência, São Paulo, v.7, n.2, p.13$22,2016$.
BARATA, L. F.; BRANCO, A.. Os distúrbios fonoarticulatórios na Síndrome de Down e a intervenção precoce. Revista CEFAC, v.12, n.1, p.134-139, 2010.

BOTELHO, F. A.; BOUZADA, M. C. F.; RESENDE, L. M.; SILVA, C. F. X. C. A.; OLIVEIRA, E. A.. Triagem auditiva em neonatos. Revista Médica de Minas Gerais, Belo Horizonte, v.18, n.4, p.139-145, 2008.

BRASIL. Ministério da Saúde. Atenção Humanizada ao Recém-Nascido de Baixo Peso: Método Canguru. 2 ed. Brasília: MS, 2011.

BRASIL. Ministério da Saúde. Diretrizes da estimulação precoce: crianças de zero a 3 anos com atraso no desenvolvimento neuropsicomotor. Brasília: MS, 2016.

BRASIL. Ministério da Saúde. Diretrizes de atenção à saúde ocular na infância: detecção e intervenção precoce para prevenção de deficiências visuais. Brasília: MS, 2013.

BRITTO, I. T.. Os bebês prematuros: concepções de suas mães acerca da intervenção fisioterapêutica. Revista InterScientia, João Pessoa, v.1, n.2, p.100-111, 2013.

CAMARGO C. C. S.; PEREIRA, S. A.; MORAN, C. A.. Estimulação sensório-motora em unidade de terapia 
intensiva neonatal: efeitos e técnicas. Revista Eletrônica Saúde e Ciência, v.7, n.2, p.62-68, 2017.

CASALI, R. L.; SANTOS, M. F. C.. Auditory Brainstem Evoked Response: response patterns of fullterm and premature infants. Brazilian Journal of Otorhinolaryngology, v.76, n.6, p.729-738, 2010. DOI: http://doi.org/10.1590/S180886942010000600011

CASTRO, G. G.; FIGUEIREDO, G. L. A.; CANO, M. A. T.. Crianças prematuras: caracterização e intervenção fisioterapêutica. Cinergis, Santa Cruz do Sul, v.17, n.3, p.196201, 2016. DOI: http://doi.org/10.17058/cinergis.v17i3.8069

DUARTE, D. T. R.; VANZO, L. C.; COPPO, M. R. C.; STOPGLIA, $M$. S.. Estimulação sensório-motora no recém-nascido. In: SARMENTO, G. J. V.. Fisioterapia respiratória em pediatria e neonatologia. 2 ed. Barueri: Manole, 2011. p.340-360.

EBSERH. Empresa Brasileira de Serviços Hospitalares. POP: Técnicas de Fisioterapia Respiratória no RN e na Criança. Uberaba: EBSERH, 2016.

FERREIRA, A. P. A.; AlBUQUERQUE, R. C.; RABELO, A. P. M.; FARIAS, F. C.; CORREIA, R. C. B.; GAGLIARDO, H. G. R. G.; LIMA, A. C. V. M. S.. Comportamento visual e desenvolvimento motor de recém-nascidos prematuros no primeiro mês de vida. Revista Brasileira de Crescimento Desenvolvimento Humano, São Paulo, v.21, n.2, p.335-43, 2011.

FIGUEIREDO, A. C.; MÜLLER, A. B.. Estimulação tátilcinestésica em bebês prematuros. Temas sobre Desenvolvimento, São Paulo, v.18, n.103, p.139-142, 2011.

FLORES, T. R.; PIGO, J. D. C.. Avaliação motora em terapia intensiva. In: LAHÓZ, A. L. C.. Fisioterapia em UTI Pediátrica e Neonatal. Barueri: Manole, 2009. p.115-124.

FORMIGA, C. K. M. R.; CEZAR, M. E. N., LINHARES, M. B. M.. Avaliação longitudinal do desenvolvimento motor e da habilidade de sentar em crianças nascidas prematuras. Revista Fisioterapia e Pesquisa, São Paulo, v.17, n.2, p.102107, 2010.

FORMIGA, C. K. M. R.; PEDRAZZANI, E. S.; TUDELA, E.. Intervenção precoce com bebês de risco. Rio de Janeiro: Atheneu, 2010.

FORMIGA, C. K. M. R.; RAMOS, B. A.. Programas de Intervenção Precoce: orientações gerais e experiências. Revista Diálogos e Perspectivas em Educação Especial, Marília, v.3, n.2, p.111-116, 2016.

GIACHETTA, L.; NICOLAU, C. M.; COSTA, A. P. B. M.; ZUANA, A. D.. Influência do tempo de hospitalização sobre o desenvolvimento neuromotor de recém-nascidos pré-termo. Fisioterapia e Pesquisa, São Paulo, v.17, n.1, p.24-29, 2010.

GOMES, N. R. R.; MONTEIRO, R. C. S.. As implicações do uso da 'redinha' por bebês prematuros: Uma revisão de literatura. Revista Ciência \& Saúde, Porto Alegre, v.16, n.2, p.94-97, 2014.

GONÇALVES, M. D. C. P.; SOARES, T. D. C.; SANTANA, L. M.. Efeitos da fisioterapia no desenvolvimento motor do lactente com deficiência visual total. Revista Eletrônica Estácio Saúde, Florianópolis, v.3, n.1, p.36-41, 2014.
JESUS, A. C.; SANTOS, A. P. M.; FERNANDES, C. R. A.. Recursos de fisioterapia respiratória. In: LAHÓZ, A. L. C... Fisioterapia em UTI pediátrica e neonatal. Barueri: Manole, 2009. p.83-102.

LINO, L. H.; COELHO, P. G.; FONSECA, F. L. A.; FILIPINI, R.. Os benefícios da rede de balanço em incubadoras utilizadas em recém-nascidos na UTI neonatal: uma estratégia de humanização. Revista de Enfermagem, Belo Horizonte, v.18, n.1, p.88-100, 2015

MEDEIROS, J. K. B.; ZANIN, R. O.; ALVES, K. S.. Perfil do desenvolvimento motor do prematuro atendido pela Fisioterapia. Revista Brasileira de Clínica Médica, São Paulo, v.7, n.6, p.367-372, 2009.

MORAN, C. A.; CACHO, R. O.; CACHO, E. W. A.; SOUSA, K. G.; SOUZA, J. C.; FILHO, G. G. F.; PEREIRA, S. A.. Use of music during physical therapy intervention in a neonatal intensive care unit: a randomized controlled trial. Journal of Human Growth and Development, v.25, n.2, p.177-181, 2015.

NICOLAU, C. M.; GIACHETTA, L.. Recursos de fisioterapia para o recém-nascido com atraso do desenvolvimento neuropsicomotor. IN. LARÓZ, A. L. C.. Fisioterapia em UTI pediátrica e neonatal. Barueri: Manole, 2009. p.141-54.

NUNES, K. S.. Estimulação tátil-cinestésica no desenvolvimento e ganho de peso do RN pré-termo na UTI. Monografia (Especialização em Pediatria e Neonatologia) Atualiza Cursos, Salvador, 2015.

OLIVEIRA, L. D.; PERUZZOLO, D. L.; SOUZA, A. P. R. Intervenção Precoce em um Caso de Prematuridade e Risco ao Desenvolvimento: Contribuições da Proposta de Terapeuta Único sustentado na interdisciplinariedade. Distúrbios da Comunicação, São Paulo, v.25, n.2, p.87-202, 2013.

OLIVEIRA, M. F.; OSELAME, G. B.; NEVES, E. B.; OLIVEIRA, E. M.. Musicoterapia como ferramenta terapêutica no setor da saúde: Uma revisão sistemática. Revista da Universidade Vale do Rio Verde, Três Corações, v.12, n.2, p.871-878, 2014.

OLMEDO, M. D.; GABAS, G. S.; MEREY, L. S. F.; SOUZA, L. S.; MULLER, K. T. C.; SANTOS, M. L. M.; MARQUES, C. F.. Respostas fisiológicas de recém-nascidos pré-termo submetidos ao Método Mãe-Canguru e a posição prona. Revista Fisioterapia e Pesquisa, São Paulo, v.19, n.2, p.115121, 2012.

PEIXOTO, P. V.; BALBINO F. S.; CHIMIRRI, V.; PINHEIRO E. M.; KAKEHASHI, T. Y.. Ruído no interior das incubadoras em unidade de terapia intensiva neonatal. Acta Paulista de Enfermagem, São Paulo, v.24, n.3, p.359-364, 2011.

PEREIRA, S. A; MORAN, C. A.. Estimulação visual precoce para recém-nascidos prematuros internados na unidade neonatal. Pediatria Moderna, São Paulo, v.8, p.358-362, 2014.

PICKLER, R. H.; MCGRATH, J. M.; REYNA, B. A; TUBBSCOOLEY, H. L.; BEST, A. I. M.; LEWIS, M.; CONE, S.; WETZEL, $P$. A.. Effects of the neonatal intensive care unit environment on preterm infant oral feeding. Research and Reports in Neonatology, v.3, p.15-20, 2013. 
PINHEIRO, A. R.; CHRISTOFOLETTI, G.. Fisioterapia motora em pacientes internados na unidade de terapia intensiva: uma revisão sistemática. Revista Brasileira de Terapia Intensiva, São Paulo, v.24, n.2, p.188-196, 2012.

PINTO, L. V.; BROCK, B.; CANTO, F.; FIGUEIREDO, H. M.; CRAMER, M.; ALMEIDA, C. S.. Intervenção motora precoce em neonatos prematuros nascidos em um hospital escola. In: SALÃO DE INICIAÇÃO CIENTÍFICA, 9. Anais. Porto Alegre: PUCRS, 2017.

PINTO, M.; SILVA, C. F. G.; MUNARI, M. M.; ALMEIDA, C. S.; RESENDE, T. L.. Intervenção motora precoce em neonatos prematuros. Revista da Graduação: Publicações de TCC, Natal, v.1, n.2, p.1-10, 2008.

POGGIOLI, M.; MINICHILLI, F.; BONONI, T.; MEGHI, P.; ANDRE, P.; CRECCHI, A.; ROSSI, B.; CARBONCINI, M. C.; OTTOLINI, A.; BONFIGLIO, L.. Effects of a home-based familycentred early habilitation program on neurobehavioural outcomes of very preterm born infants: a retrospective cohort study. Revista Neural Plasticity, p.1-10, 2016.

RAMADA, N. C. O.; ALMEIDA, F. A.; ROCHA, M. L.. Toque terapêutico: influência nos parâmetros vitais de recémnascidos. Einstein, São Paulo, v.11, n.4, p.421-425, 2013.

RAMOS, H. A. C.; CUMAM, R. K. N.. Fatores de risco para prematuridade: pesquisa documental. Escola Anna Nery Revista de Enfermagem, Rio de Janeiro, v.13, n.2, p.297-304, 2009.

SCHAEFER, M. P.; DONELLI, T. M. S.; MARIN, A. H.. Pesquisa e intervenção no contexto da prematuridade: considerações metodológicas. Revista Investigação Qualitativa em saúde, Rio de Janeiro, v.6, n.2, p.472-481, 2017.

SEGURA, D. C. A.; NACIMENTO, F. C.; CALABRESI, M. C. B.; CÉRON, L. B.. Indicação da Fisioterapia ambulatorial para neonatos pré-termo. Revista Saúde e Pesquisa, Maringá, v.3, n.3, p.321-327, 2010.

SILVA, A. R. E.; GARCIA, P. N.; GUARIGLIA, D. A.. Método Canguru e os benefícios para o recém-nascido. Revista Hórus, Ourinhos, v.8, n.2, p.1-10, 2013.

SILVA, C. C. V.. Atuação da fisioterapia através da estimulação precoce em bebês prematuros. Atualiza Saúde, Salvador, v.5, n.5, p.29-36, 2017.
SILVA, C. M.; CAÇÃO, J. M. R.; SILVA, K.C.S.; MARQUES, C. F.; MEREY, L. S. F.. Respostas fisiológicas de recém-nascidos pré-termo submetidos à musicoterapia clássica. Revista Paulista de Pediatria, São Paulo, v.31, n.1, p.30-36, 2013.

SILVA, H. A.; SILVA, K. C.; RECO, M. O. N.; COSTA, A. S.; MARANGONI, D. A. S.; MEREY, L. S.. Efeitos fisiológicos da hidroterapia em balde em recém-nascidos prematuros. Revista de Terapia Ocupacional da Universidade de São Paulo, São Paulo, v.28, n.3, p.309-315, 2017. DOI: http://doi.org/10.11606/issn.2238-6149.v28i3p309-315

SILVA, S. B.; SANTOS L. M.; BERREIRA, F. S.; ALMEIDA, C. D. A.. Estimulação precoce em bebês prematuros. In: CONGRESSO BRASILEIRO DE CIÊNCIAS DA SAÚDE, 2. Anais. Campina Grande: Centro de Convenções, 2017.

SOUZA, K. C. L.; CAMPOS, N. G.; SANTOS JÚNIOR, F. F. U.. Perfil dos recém-nascidos submetidos à estimulação precoce em uma unidade de terapia intensiva neonatal. Revista Brasileira em Promoção da Saúde, Fortaleza, v.26, n.4, p.523-529, 2013.

SOUZA, T. A; SOUZA, V. E.; LOPES, M. C. B.; KITADA, S. P. S.. Descrição do desenvolvimento neuropsicomotor e visual de crianças com deficiência visual. Arquivos Brasileiros de Oftalmologia, São Paulo, v.73, n.6, p.526-530, 2010.

THEIS, R. C. S. R.; GERZON, L. R. G.; ALMEIDA, C. S.. A atuação do profissional fisioterapeuta em unidades de terapia intensiva neonatal. Cinergis, Santa Cruz do Sul, v.17, n.2, p.168-176, 2016. DOI:

http://dx.doi.org/10.17058/cinergis.v17i2.7703.

TOSO, B. R. G. O; VIERA, C. S.; VALTER, J. M.; DELATORE, S.; BARRETO, G. M. S.. Validação de protocolo de posicionamento de recém-nascido em Unidade de Terapia Intensiva. Revista Brasileira de Enfermagem, Brasília, v. 68, n. 6, p. 835-41, 2015.

URZÊDA, R. N.; OLIVEIRA, T. G.; CAMPOS, A. M.; FORMIGA, C. B. M. R.. Reflexos, reações e tônus muscular de bebês prétermo em um programa de intervenção precoce. Revista Neurociências, São Paulo, v.17, n.4, p.319-25, 2009.

VIGNOCHI, C.; TEIXEIRA, P. P.; NADER, S. S.. Efeitos da fisioterapia aquática na dor e no estado de sono e vigília de recém-nascidos pré-termo estáveis internados em unidade de terapia intensiva neonatal. Revista Brasileira de Fisioterapia, São Carlos, v.14, n.3, p.214-220, 2010.

A CBPC - Companhia Brasileira de Produção Científica (CNPJ: 11.221.422/0001-03) detém os direitos materiais desta publicação. Os direitos referem-se à publicação do trabalho em qualquer parte do mundo, incluindo os direitos às renovações, expansões e disseminações da contribuição, bem como outros direitos subsidiários. Todos os trabalhos publicados eletronicamente poderão posteriormente ser publicados em coletâneas impressas sob coordenação da Sustenere Publishing, da Companhia Brasileira de Produção Científica e seus parceiros autorizados. Os (as) autores (as) preservam os direitos autorais, mas não têm permissão para a publicação da contribuição em outro meio, impresso ou digital, em português ou em tradução. 